\title{
Microbiological and parasitological survey of zoonotic agents in apparently healthy feral pigeons
}

\author{
M.L. Marenzoni' ${ }^{1}$, G. Morganti ${ }^{1}$, I. Moretta ${ }^{1}$, S. Crotti ${ }^{2}$, F. Agnetti ${ }^{2}$, A. Moretti ${ }^{1}$, \\ L. Pitzurra ${ }^{3}$, P. Casagrande Proietti ${ }^{1}$, P. Sechi ${ }^{1}$, B. Cenci-Goga ${ }^{1}$, \\ M.P. Franciosini ${ }^{1}$ \\ ${ }^{1}$ Dipartimento di Medicina Veterinaria, Via San Costanzo 4, 06126, Perugia, Italy \\ ${ }^{2}$ Istituto Zooprofilattico Sperimentale Umbria e Marche, Via G. Salvemini 1, 06126, Perugia, Italy \\ ${ }^{3}$ Dipartimento di Medicina Sperimentale e Scienze Biochimiche, Ospedale Santa Maria della Misericordia, \\ Piazzale Menghini 1, 06156, Perugia, Italy
}

\begin{abstract}
Microbiological and parasitological investigation was carried out on a colony of feral pigeons, located in a green area near the main hospital of a Central Italy city. One hundred pigeons were submitted to clinical examination. Cloacal swabs, grouped in pool of 4 samples, were analyzed to detect the presence of Coxiella burnetii, Chlamydia psittaci, Chlamydophila spp. using a biomolecular procedure, while individual cloacal samples were examined for Salmonella spp., Campylobacter spp., and yeasts by means of a specific culture media. An ELISA test was used to determine the presence of Giardia spp., and Cryptosporidium spp. coproantigens. Individual serological samples were also tested with the modified agglutination test (MAT) in order to detect antibodies against Toxoplasma gondii. The pigeons did not show any clinical signs. The cloacal pools proved to be negative for $C$. burnetii DNA while three pools were positive for C. psittaci or Chlamydophila spp. DNAs. Salmonella spp. was not detected. C. jejuni and C. coli were found in $13 \%$ and $4 \%$ of the samples, respectively. No Giardia spp. and Cryptosporidium spp. were detected. Thirty-three out of 100 samples (33\%) were positive for yeast colonies. The seroprevalence for $T$. gondii was $8 \%$. Although with moderate incidence, potentially zoonotic agents were present thus highlighting the need for sanitary surveillance on feral pigeon colonies.
\end{abstract}

Key words: feral pigeon, zoonotic agent, hospital, public health 


\section{Introduction}

Nowadays feral pigeons can be found all over the world interacting with human beings and their activities in several ways. Pigeons appear to be harmless yet they become a threat for Public Health (Jerolmack 2008). At present, approximately several hundred million feral pigeons are estimated to live in cities worldwide and the size of their colonies mainly depends on the amount of food provided by humans and on natural seasonal foodstuffs to a lesser extent (Haag-Wackernagel 2011). In some cases, the increase in number of these populations can cause concern as they frequently colonize squares, public parks or areas, where people at risk, such as children, the elderly or people with depressed immune systems, may inadvertently come into contact with their excreta in the form of aerosolized particles (Haag-Wackernagel and Moch 2004). In this context, feral pigeons have a significant epidemiological role, since they can be reservoirs and potential vectors of a large number of zoonotic pathogens as well as responsible for allergic diseases (Haag-Wackernagel and Bircher 2009, Magnino et al. 2009). Alexander et al. (1985) described approximately 110 zoonotic agents isolated from pigeons up to now. Salmonella spp., Campylobacter spp., Shiga toxin-producing, E. coli, chlamydial agents and Cryptococcus spp. are carried by clinically healthy pigeons (Haag-Wackernagel and Moch 2004, Pedersen et al. 2006, Kobayashi et al. 2007, Sachse et al. 2012). However, the association between human practice and illnesses contracted from feral pigeons has seldom been reported (Haag-Wackernagel and Moch 2004). Cryptococcus neoformans, Candida albicans, and Chlamydophila psittaci seem to be the most widespread zoonotic agents in the pigeon populations that reside in cities worldwide (Heddema et al. 2006, Magnino et al. 2009, Tomić et al. 2013). Epidemiological surveys for $C$. psittaci carried out on pigeons across Europe during the last thirty years detected high seropositivity and high prevalence in feces (Heddema et al. 2006, Magnino et al. 2009, Vazquez et al. 2010), although differences were observed among the diagnostic procedures used and the epidemiological conditions found in various studies. However, the presence of zoonotic organisms in feral pigeons does not necessarily cause disease in man, since several other factors such as the transmission route, the infectious dose, the immune status of the people involved and the closeness of contact strongly influence the possibility of infection (Haag-Wackernagel and Moch 2004). The aim of this study was to carry out a microbiological and parasitological survey on a colony of feral pigeons located near the main hospital situated in the suburbs of Perugia, a city of Central Italy, in order to assess the potential pathogen risk of transmission to both healthy and immunocompromised populations.

\section{Materials and Methods}

One hundred pigeons were captured with nets from a colony of approximately 200 feral pigeons, located near the main hospital in the suburbs of Perugia. After clinical examination, cloacal swabs and blood samples were collected in order to determine the presence of the following zoonotic agents: Coxiella burnetii, Chlamydophila psittaci, Chlamydophila spp., Salmonella spp., Campylobacter spp., Giardia spp., Cryptosporidium spp., T. gondii and yeasts.

Biomolecular investigation was performed to detect C. burnetii, C. psittaci and Chlamydophila spp.. Twenty-five cloacal pools (no. 1 to 25) were obtained by mixing 4 individual samples and their DNAs were extracted from $200 \mathrm{fl}$ of each pool with a commercial kit (QIAamp DNA Stool mini kit, Qiagen, Italy) according to the manufacturer's instructions. A PCR protocol described by Berri et al. (2000), which involves amplifying a fragment of $687 \mathrm{bp}$ of the IS1111 insertion sequence, was used with modifications. Two different nested PCR assays were used to identify the Chlamydophila spp. genus and $C$. psittaci species. Briefly, the Chlamydophila genus was recognized in the first step of the protocol described by Messmer et al. (1997) which targets the 16S rRNA gene, while the $C$. psittaci species was identified by both the nested step of the protocol by Messmer et al. (1997) and the amplification of the ompA gene (Van Look 2005). The DNA of each sample was tested in duplicate. The primers and cycling conditions are described in Table 1. Positive and negative DNA controls were used for each set of reactions. The PCR products of the expected size were purified by means of a gel extraction kit (Qiaquick PCR purification kit, Qiagen, Italy) and directly sequenced on both strands with the same primers using DNA analyzer (ABI 3730, Applied Biosystems) capillary sequencer (Primm Srl) as previously described. The sequences were assembled and aligned using BioEdit (Biological sequence alignment editor software, version 7.0.8). The sequence similarity was checked against sequences deposited in GenBank using BLAST (Basic local alignment search) software to confirm the species specificity of the PCR. 


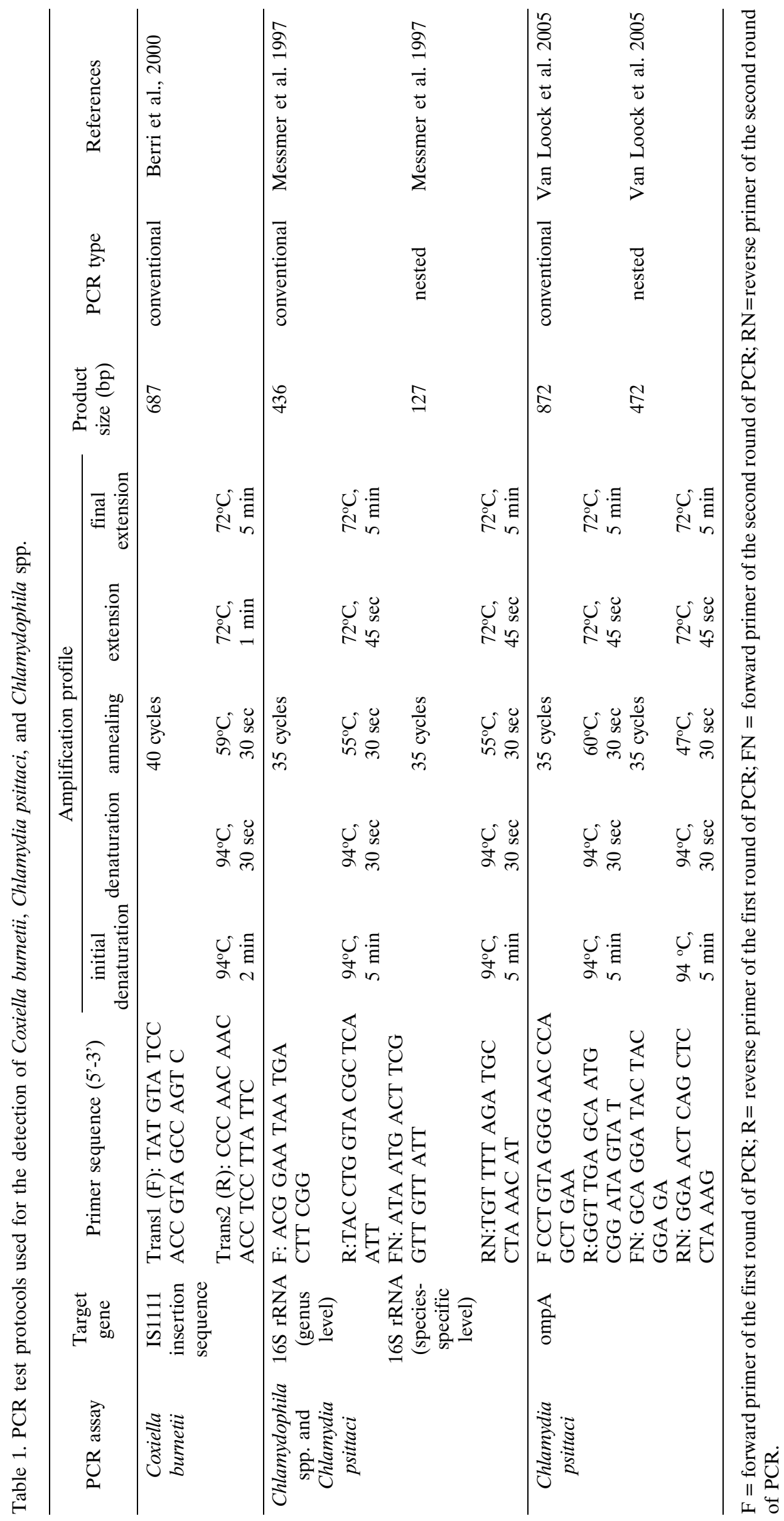


Table 2. Yeast identification by biochemical test and by MALDI TOF MS.

\begin{tabular}{lclc}
\hline \multicolumn{1}{c}{ Biochemical identification } & \multicolumn{1}{c}{ Maldi-tof ms identification } \\
\hline \multicolumn{1}{c}{ Yeast species } & No. isolates & \multicolumn{1}{c}{ Yeast species } & No. isolates \\
\hline Candida zeylanoides & 2 & C. zeylanoides & 2 \\
Candida famata & 5 & C. guillermondi & 5 \\
Candida incospicua & 1 & C. incospicua & 1 \\
Candida lusitaniae & 3 & C. lusitaniae & 1 \\
Cryptococcus albidus & 4 & C. albidus & 1 \\
Rhodotorula mucilaginosa & 3 & R. mucilaginosa & 3 \\
Rhodotorula glutinis & 1 & R. mucilaginosa & 1 \\
Zygosaccharomyces spp. & 14 & C. krusei & 4 \\
Total & 33 & Total & 18 \\
\hline
\end{tabular}

The enrichment medium, Rappaport-Vassiliadis broth, was used for detecting Salmonella spp. The samples were subcultured at $37^{\circ} \mathrm{C}$ on xylose lysine desoxycholate agar (XLD, Oxoid, Italy) and brilliant green agar (Oxoid, Italy) solid selective media. The results were read after 24 and $48 \mathrm{~h}$ of incubation. Suspected colonies were identified by means of composite biochemical media and commercial biochemical tests such as API RAPID 20E (BioMerieux, Italy). Cloacal specimens were analyzed to detect Campylobacter spp. diluted in Maximum Recovery Diluent (MRD, Oxoid, Italy) and vortexed until dissolution. The samples were surface spread-plated in duplicates onto dried, modified Cefaperazone Charcoal Desoxycholate Agar (mCCDA, Oxoid, Italy) plates, that were incubated micro aerobically for 48 h at $41.5^{\circ} \mathrm{C}$. The presence of Campylobacter spp. was confirmed by the oxidase activity, absence of growth in $\mathrm{O}_{2}$ and agglutination test (Microgen ${ }^{\circledR}$ Campy Latex Agglutination Kit, Microgen Bioproduct, United Kingdom).

Giardia spp. and Cryptosporidium spp. coproantigens were investigated using individual cloacal samples which were then submitted to ELISA test (ProSpecT, Oxoid, Italy), according to the manufacturer's instructions. Individual cloacal swabs were cultured in Sabouraud Dextrose Agar, incubated at $24 \pm 1^{\circ} \mathrm{C}$ in aerobiosis and observed on a daily basis in order to monitor the macro and microscopic characteristics of the yeast growth. The yeasts were subsequently identified with biochemical tests (API ID32C, BioMérieux, Italy); contemporarily a more feasible and specific method based on MALDI-TOF MS (Matrix-Assisted Laser Desorption/Ionization Time-of-Flight Mass Spectrometry) was used in order to confirm the biochemical identification of the isolates. In the event of discordant results, MALDI-TOF MS was used to obtain definitive identification.

Serum samples were tested for $T$. gondii antibodies by means of the MAT (modified agglutination test), as described previously (Yan et al. 2011). In accordance with a recent study (Dubey 2010), MAT serum titers of 1:5 or higher were considered positive.

\section{Results}

The pigeons were well fed and did not show any clinical signs.

All cloacal pools were negative for $C$. burnetii DNAs, while one (no. 3) proved to be positive for $C$. psittaci DNA with both nested PCR protocols. Pool numbers 8 and 12 were only positive for the presence of gene 16S rRNA at the nested step. Sequencing of the ompA amplicon of pool no. 3 (GenBank accession no. KP704660) confirmed it as being C. psittaci at $99-100 \%$ homology with the sequences reported in GenBank. The sequencing of the amplicons, obtained from the three pools positive to the $16 \mathrm{~S}$ rRNA gene (nos. 3,8 , and 12), identified various chlamydial species such as $C$. psittaci, C. abortus, C. avium, $C$. felis, C. muridarum, C. pecorum, C. trachomatis, although the nested step was considered species-specific for $C$. psittaci. For this reason, only pool no. 3 was identified as $C$. psittaci DNA and proved to be positive with both nested PCR protocols which was confirmed through sequencing, while the other two (nos. 8 and 12) probably contained DNA of various chlamydial species.

Salmonella spp. was not detected. C. jejuni and C. coli were found in $13 \%$ and $4 \%$ (positives/total subjects) of cases, respectively.

No Giardia spp. and Cryptosporidium spp. coproantigens were detected.

Thirty-three out of 100 samples were positive for yeast colonies at macro and micro features. Isolated yeast species with different tools are reported in Table 2.

The overall seroprevalence of $T$. gondii was $8 \%$ with the 1:10 end-point titre. 


\section{Discussion}

In this study the positivity for $C$. psittaci DNA proved that pigeons are possible carriers of the infection in the investigated area, although it is impossible to estimate to what extent since the results were obtained from pool samples. However, the positive results were relevant as the pigeons did not show any clinical signs, in accordance with data reported by Vazquez et al. (2010), thus supporting the hypothesis that they may act as asymptomatic reservoirs of $C$. psittaci or other chlamydial species. Moreover, in the absence of clinical signs, the shedding of chlamydia is generally intermittent making it difficult to detect (Tomić et al. 2013). Nevertheless, there is a remarkable discrepancy between the widespread distribution of this pathogen in pigeon populations and the low number of human cases reported (Haag-Wackernagel and Moch 2004, Magnino et al. 2009), although it is possible that they may be underestimated due to diagnostic difficulties and the variability of clinical signs. Moreover, several authors have suggested that pigeon-derived $C$. psittaci strains have a low pathogenicity for man (Magnino et al. 2009). Lastly, pigeons could be also vectors of other chlamydial species that are relevant for their zoonotic role as previously reported (Sachse et al. 2012), which was confirmed in this study due to the detection of DNAs of various chlamydial species. However, further characterization of these sequences and an in-depth study of their zoonotic and epidemiological role are required. In this study $C$. burnetii DNA was not detected. Pigeons had previously been reported as being responsible for Q fever outbreaks in humans (Stein and Raoult 1999). In our samples $C$. jejuni and C. coli were found in $13 \%$ and $4 \%$ of the cases, respectively. Casanovas et al. (1995) demonstrated that C. jejuni was the most frequently isolated bacterium in 105 pigeons $(26.2 \%)$ with a greater incidence in the districts of the city of Barcelona regardless of seasonal variation. Vazquez et al. (2010) also detected a high prevalence of $C$. jejuni and low prevalence of $C$. coli, as in our study. However, despite the frequent isolation of Campylobacter spp. from pigeons, direct transmission to humans has not yet been reported (Haag-Wackernagel and Moch 2004). On the basis of their studies regarding the adhesion and invasion models, Teske et al. (2013) suggested that pigeon-derived $C$. jejuni isolates were capable of infecting human enterocytes and producing the virulence factor cytolethal-distending toxin.

In this study Salmonella spp. was not detected thus confirming the observations of previous studies in which the isolation of Salmonella in feral pigeons was generally low (Pedersen et al. 2006). Similar re- sults were obtained in a previous study, which only reported a single isolate of S. typhimurium in 202 pigeons monitored in the center of Perugia (Maresca et al. 2011). A review of health hazards caused by feral pigeons reported one single case of $S$. enterica serotype Kiambu infection in man (Haag Wackemagel and Moch 2004). In a two-year study carried out on racing pigeons, Teske et al. (2013) reported a range from a minimum of 0.9 to $3.7 \%$ of the positive lots investigated. In older studies (Vindevogel and Duchatel, 1979) the incidence of Salmonella spp. reached $26 \%$ in diseased pigeons submitted for diagnostic purposes. However, only clinically healthy pigeons were included in the present study. In this type of scenario, the incidence of Salmonella spp. is generally low and it is seldom transmitted from feral pigeon to man.

Concerning parasitological investigations, our samples proved to be negative for enteric protozoa while several studies have detected $C$. parvum and $C$. hominis, and the zoonotic assemblages of $G$. duodenalis (A and $\mathrm{B}$ ) in faeces of various avian species indicating that birds might serve as mechanical vectors of cysts and oocysts and as possible sources of these infections in animals and humans (Graczyk et al. 2008, Abreu-Acosta et al. 2009, Majewska et al. 2009, Papini et al. 2012, Reboredo-Fernandez et al. 2015). At present, little information is available concerning the role played by pigeons in the zoonotic transmission of these pathogens. Probably, giardiasis and cryptosporidiosis spread more easily in overcrowded environments or stressful conditions, i.e. animals kept in zoos (Papini et al. 2012), than in small or medium-sized colonies like these examined in our study.

In our investigation, the overall seroprevalence of T. gondii (8\%) was in accordance with data previously obtained in Italy (Grandini and Sarti 1995) even if a more recent report carried out in colonies of pigeons in Central Italy showed a seroprevalence of 83\% (Maresca et al. 2011). Moreover, contrasting percentages of prevalence ranging from $4 \%$ to $100 \%$ can also be found in international literature due to differences in geographical conditions, diet and lifestyle, number of cats and rodents, as well as the serological test used (Mushi et al. 2001, Salant et al. 2009, Karatepe et al. 2011, Cong et al. 2014). Considering that pigeons share the environment with humans, the serological results suggest that they could be a good model and an indicator of the extent of oocyst contamination of the ground. Moreover, they can serve as sources of tissue cysts for domestic felids thus contributing to the epidemiological circuit of $T$. gondii (Karatepe et al. 2011).

In this study the pigeons frequently harbored a mixed potentially pathogenic fungi flora in the gas- 
trointestinal tract. Although yeast has been considered a commensal organism and part of the normal biota of pigeons, over the last decade, Cryptococcus albidus and non-Candida albicans species, which were also isolated in this study, have emerged in immunocompromised and hospitalized patients (Dorneanu et al. 2008, Beyda et al. 2013). Recent studies on outbreaks of Candida species showed an increased incidence of bloodstream infections caused by $C$. parapsilosis in neonatal intensive care units (Clarck et al. 2013). Other yeasts, such as Rhodotorula and Trichosporon, can cause blood infections in patients with indwelling central venous catheters and cancer (Cafarchia et al. 2006). Several studies have confirmed the role of pigeon in the epidemiology of these opportunistic pathogen agents causing human infection due to inhalation or contact with their dried feces (Andrade-Silva et al. 2010, Rosario et al. 2010). In the present work, not all isolates of yeasts evidenced by biochemical test were confirmed and/or detected by MALDI TOF MS. Despite the specificity of the tool, Clark et al. (2013) evidenced that data-bases of MALDI TOF MS are not developed for several yeast species and this could justify the lower number of isolates identified. However, the detection of several zoonotic yeasts from pigeons located in urban areas can provide useful preliminary information for epidemiological and risk assessment studies.

In conclusion, proper control of the feral pigeon population and careful monitoring for zoonotic agents should be always carried out in areas, such as parks, hospitals, and schools, where subjects with impaired or not completely developed immune systems could be present (Dolbeer et al. 2000).

\section{References}

Abreu-Acosta N, Foronda-Rodriguez P, Lopez M, Valladares B (2009) Occurrence of Cryptosporidium hominis in pigeons (Columbia livia). Acta Parasitol 54: 1-5.

Alexander DJ, Wilson GW, Russell PH, Lister SA, Parsons $G$ (1985) Newcastle disease outbreaks in fowl in Great Britain during 1984. Vet Rec 117: 429-434.

Andrade-Silva L, Ferreira-Paim K, Silva-Vergara ML, Pedrosa AL (2010) Molecular characterization and evaluation of virulence factors of Cryptococcus laurentii and Cryptococcus neoformans strains isolated from external hospital areas. Fungal Biol 114: 438-445.

Berri M, Laroucau K, Rodolakis A (2000) The detection of Coxiella burnetii from ovine genital swabs, milk and fecal samples by the use of a single touchdown polymerase chain reaction. Vet Microbiol 72: 285-293.

Beyda ND, Chuang SH, Alam MJ, Shah DN, Ng TM, McCaskey L, Garey KW (2013) Treatment of Candida famata bloodstream infections: case series and review of the literature. J Antimicrob Chemother 68: 438-443.

Cafarchia C, Camarda A, Romito D, Campolo M, Quaglia
NC, Tullio D, Otranto D (2006) Occurrence of yeasts in cloacae of migratory birds. Mycopathologia 161: 229-234.

Casanovas L, De Simon M, Ferrer MD, Arques J, Monzon $G$ (1995) Intestinal carriage of campylobacters, salmonellas, yersinias and listerias in pigeons in the city of Barcelona. J Appl Bacteriol 78: 11-13.

Clark AE, Kaleta EJ, Arora A, Wolk DM (2013) Matrix-Assisted Laser Desorption Ionization-Time of Flight Mass Spectrometry: a Fundamental Shift in the Routine Practice of Clinical Microbiology. Clin Microbiol Rev 26: 547-603.

Cong W, Meng QF, Song HQ, Zhou DH, Huang SY, Qian $\mathrm{AD}, \mathrm{Su} \mathrm{C}, \mathrm{Zhu} \mathrm{XQ}$ (2014) Seroprevalence and genetic characterization of Toxoplasma gondii in three species of pet birds in China. Parasit Vectors 7: 152.

Dolbeer RA, Wright SE, Cleary EC (2000) Ranking the hazard level of wildlife species to aviation. Wildlife Soc Bull 28: 372-378.

Dorneanu O, Filip O, Miftode E, Radu I, Nicolau C, Damian I, Luca V (2008) Cryptococcus meningitis, five years of experience and literature review. Rev Med Chir Soc Med Nat Iasi 112: 100-103.

Dubey JP (2010) Toxoplasma gondii infections in chickens (Gallus domesticus): prevalence, clinical disease, diagnosis and public health significance. Zoonoses Public Health 57: 60-73.

Graczyk TK, Majewska AC, Schwab KJ (2008) The role of birds in dissemination of human waterborne enteropathogens. Trend Parasitol 24: 55-59.

Grandini S, Sarti E (1995) Controllo dei piccioni in ambiente urbano. O\&DV 12: 57-60 [Article in Italian]

Haag-Wackernagel D (2011) Vom Liebling der Gotter zur Eroberunder Stadte Die Taube-eine Erfolgsgeschichte. Biol. Unserer Zeit 41: 44-52. [Article in German]

Haag-Wackernagel D, Bircher AJ (2009) Ectoparasites from feral pigeons affecting humans. Dermatology 220: 89-92.

Haag-Wackernagel D, Moch H (2004) Health hazards posed by feral pigeons. J Infect 48: 307-313.

Heddema ER, Ter Sluis S, Buys JA, Vandenbroucke-Grauls CM, van Wijnen JH, Visser CE (2006) Prevalence of Chlamydophila psittaci in fecal droppings from feral pigeons in Amsterdam, The Netherlands. Appl Environ Microbiol 72: 4423-4425.

Jerolmack C (2008) How pigeons became rats: the cultural-spatial logic of problem animals. Social Problems 55: 72-94.

Karatepe M, Kulıç S, Karatepe B, Babür C (2011) Prevalence of Toxoplasma gondii antibodies in domestic (Columba livia domestica) and wild (Columba livia livia) pigeons in Niede region, Turkey. Turkiye Parazitol Derg 35: 23-26.

Kobayashi H, Kanazaki M, Shimizu Y, Nakajima H, Khatun M, Hata E, Kubo M (2007) Salmonella isolate from cloacal swabs and footpads of wild birds in the immediate environment of Tokyo Bay. J Med Vet Sci 69: 309-311.

Magnino S, Haag-Wackernagel D, Geigenfeind I, Helmecke S, Dovc A, Prukner-Radovcic E, Residbegovic E, Ilieski V, Laroucau K, Donati M, Martinov S, Kaleta EF (2009) Chlamydial infections in feral pigeons in Europe: Review of data and focus on public health implications. Vet Microbiol 135: 54-67.

Majewska AC, Graczyk TK, Słodkowicz-Kowalska A, Tamang L, Jedrzejewski S, Zduniak P, Solarczyk P, 
Nowosad A, Nowosad P (2009) The role of free-ranging, captive, and domestic birds of Western Poland in environmental contamination with Cryptosporidium parvum oocysts and Giardia lamblia cysts. Parasitol Res 104: 1093-1099.

Maresca C, Scoccia E, D'Angelo G, Cristina Neri M, Caporali A, Tentellini M, Grelloni V (2011) Monitoring the health of the population of pigeons (Columba livia) in the city of Perugia (2010-2011). Sanitg Pubblica Veterinaria 69: ISSN 1592-1581.

Messmer TO, Skelton SK, Moroney JF, Daugharty H, Fields BS (1997) Application of a nested, multiplex PCR to psittacosis outbreaks. J Clin Microbiol 35: 2043-2046.

Mushi EZ, Binta MG, Chabo RG, Ndebele R, Panzirah $\mathrm{R}$ (2001) Seroprevalence of Toxoplasma gondii and Chlamydia psittaci in domestic pigeons (Columbia livia domestica) at Sebele, Gaborone, Botswana. Onderstepoort J Vet Res 68: 159-161.

Papini R, Girivetto M, Marangi M, Mancianti F, Giangaspero A (2012) Endoparasite infections in pet and zoo birds in Italy. Scientific World Journal 2012: 253127.

Pedersen K, Clark L, Andelt WF, Salman M (2006) Prevalence of Shiga toxin-producing Escherichia coli and Salmonella enterica in rock pigeons captured in Fort Collins, Colorado. J Wildl Dis 42: 46-55.

Reboredo-Fernández A, Ares-Mazás E, Cacció SM, Gómez-Couso H (2015) Occurrence of Giardia and Cryptosporidium in wild birds in Galicia (Northwest Spain). Parasitology 142: 917-925.

Rosario I, Soro G, Déniz S, Ferrer O, Acosta F, Padilla D, Acosta B (2010) Presence of C. albidus, C. laurentii and C. uniguttulatus in crop and droppings of pigeon lofts (Columba livia). Mycopathologia 169: 315-319.
Sachse K, Kuehlewind S, Ruettger A, Schubert E, Rohde G (2012) More than classical Chlamydia psittaci in urban pigeons. Vet Microbiol 157: 476-480.

Salant H, Landau DY, Baneth G (2009) A cross-sectional survey of Toxoplasma gondii antibodies in Israeli pigeons. Vet Parasitol 165: 145-149.

Stein A, Raoult D. (1999) Pigeon pneumonia in provence: a bird-borne $Q$ fever outbreak. Clin Infect Dis 29: 617-620.

Teske L, Ryll M, Rautenschlein S (2013) Epidemiological investigations on the role of clinically healthy racing pigeons as a reservoir for avian paramyxovirus- 1 and avian influenza virus. Avian Pathol 42: 557-565.

Tomić DH, Laroucau K, Prukner-Radovčić E (2013) Detection of Chlamydia psittaci genotypes in fecal samples of homing pigeons in Croatia. Vet Arhiv 83: 201-209.

Van Loock M, Verminnen K, Messmer TO, Volckaert G, Goddeeris BM, Vanrompay D (2005) Use of a nested PCR-enzyme immunoassay with an internal control to detect Chlamydophila psittaci in turkeys. BMC Infect Dis 5: 76.

Vazquez B, Esperon F, Neves E, Lopez J, Ballesteros C, Munoz MJ (2010) Screening for several potential pathogens in feral pigeons (Columba livia) in Madrid. Acta Vet Scandin 52: 45.

Vindevogel H, Duchatel JP (1979) Les principales maladies parasitaires du pigeon. Ann Med Vet 123: 85-92. [Article in French]

Yan C, Yue CL, Qiu SB, Li HL, Zhang H, Song HQ, Huang SY, Zou FC, Liao M, Zhu XQ (2011) Seroprevalence of Toxoplasma gondii infection in domestic pigeons (Columba livia) in Guangdong Province of southern China. Vet Parasitol 177: 371-373. 